\title{
Study of meteoroid stream identification methods
}

\author{
Regina Rudawska ${ }^{1}$ and Tadeusz J. Jopek ${ }^{2}$ \\ Astronomical Observatory of Adam Mickiewicz University, Poznan, Poland \\ ${ }^{1}$ email: reginka@amu.edu.pl ${ }^{2}$ email: jopek@amu.edu.pl
}

\begin{abstract}
We have tested the reliability of various meteoroid streams identification methods. We used a numerically generated set of meteoroid orbits (a stream component and a sporadic background) that were searched for streams using several methods.
\end{abstract}

Keywords. meteoroids, data analysis

\section{Introduction}

The meteoroid stream identification methods are based on three components: dynamical similarity (distance) function, similarity threshold $D_{c}$ and cluster analysis algorithm - which define the stream itself. Several similarity functions have been proposed: $D_{S H}$ the orbital D-criterion introduced by Southworth and Hawkins (1963), its modifications by Drummond (1981), Jopek (1993), Valsecchi et al. (1999), and Jenniskens (2008) and the $D_{V}$ function defined in the domain of the vectorial heliocentric orbital elements (Jopek et al. (2008)). The threshold, $D_{c}$ is used to test the similarity among two orbits $\mathbf{O}_{i}, \mathbf{O}_{j}$ - these two orbits are associated if $D\left(\mathbf{O}_{i}, \mathbf{O}_{j}\right)<D_{c}$. Having the distance function and the similarity threshold a meteoroid stream can be detected by a cluster analysis algorithm, one can use e.g.: an iterative methods proposed in (Sekanina (1976), Welch (2001)), a single neighbour linking technique (Southworth and Hawkins (1963), Lindblad (1971)), method of indices (Svoreň et al. (2000)) or the wavelet transform technique (Galligan and Baggaley (2002), Brown et al. (2008)).

\section{Meteoroid data sample preparation}

We searched for streams among the numerically generated orbits. For selected NEOs (see table 1), fifteen sets of genetically associated particles were generated; the motion of all stream particles was integrated numerically over 40KA with the Newtonian force model of the Planetary System. At each of six intermediate epochs (see table 2) the stream component was completed by a set of sporadic orbits generated with the distribution of orbital elements that were statistically similar to the distribution of the background meteors taken from the IAU MDC photographic catalogue, Lindblad et al. (2003).

\section{Searching methods tested in this study}

The orbital samples were searched by the following methods:

- $W 1 D S H$ - a simplified version of Welch's method (Welch (2001)) equipped with the $D_{S H}$ function. In this method, the density at mean orbit of a stream $\mathbf{O}_{A}$ in orbital 


\begin{tabular}{|c|c|c|c|c|c|c|c|c|c|}
\hline Parent body & $q$ & $a$ & $e$ & $i$ & $\Omega$ & $\omega$ & $Q$ & $P$ & Associated meteoroid stream \\
\hline $1998 \mathrm{SH}_{2}$ & 0.743 & 2.686 & 0.723 & 2.5 & 13.1 & 261.2 & 4.629 & 4.40 & $\alpha$ Virginids \\
\hline $2004 \mathrm{BZ}_{74}^{2}$ & 0.330 & 3.048 & 0.892 & 16.6 & 233.9 & 121.3 & 5.767 & 5.32 & $\alpha$ Scorpiids \\
\hline $2004 \mathrm{HW}^{4}$ & 0.976 & 2.688 & 0.637 & 0.8 & 220.4 & 62.3 & 4.401 & 4.41 & Corvids \\
\hline $2004 \mathrm{TG}_{10}$ & 0.315 & 2.242 & 0.859 & 3.7 & 212.3 & 310.0 & 4.169 & 3.36 & N. Taurids, Dayt. $\beta$ Taurids \\
\hline $2005 \mathrm{NZ}_{6}^{10}$ & 0.248 & 1.834 & 0.865 & 8.5 & 39.7 & 48.0 & 3.419 & 2.48 & Dayt. April Piscids \\
\hline 3200 Phaethon & 0.140 & 1.271 & 0.890 & 22.2 & 265.4 & 322.0 & 2.403 & 1.43 & Geminids \\
\hline $1 \mathrm{P} / \mathrm{H}$ alley & 0.587 & 17.942 & 0.967 & 162.2 & 58.9 & 111.9 & 35.296 & 76.00 & Orionids, $\eta$ Aquariids \\
\hline $2 \mathrm{P} /$ Encke & 0.331 & 2.209 & 0.850 & 11.9 & 334.7 & 186.2 & 4.087 & 3.28 & S. Taurids. Dayt. $\zeta$ Perseids \\
\hline $7 \mathrm{P} /$ Pons $-\mathrm{W}$ innecke & 1.256 & 3.435 & 0.634 & 22.3 & 93.4 & 172.3 & 5.615 & 6.37 & June Bootids \\
\hline $8 \mathrm{P} /$ Tuttle & 0.998 & 5.672 & 0.824 & 54.7 & 270.5 & 206.7 & 10.346 & 13.50 & Ursids \\
\hline $21 \mathrm{P} / \mathrm{G}$ iacobinni-Zimmer & 1.034 & 3.522 & 0.706 & 31.8 & 195.4 & 172.5 & 6.010 & 6.61 & Draconids \\
\hline $26 \mathrm{P} /$ Grigg-Skjellerup & 0.997 & 2.965 & 0.664 & 21.1 & 213.3 & 359.3 & 4.933 & 5.11 & $\pi$ Puppids \\
\hline $55 \mathrm{P} /$ Temple-Tuttle & 0.977 & 10.337 & 0.905 & 162.5 & 235.3 & 172.5 & 19.698 & 3.28 & Leonids \\
\hline $73 \mathrm{P} / \mathrm{Sch}$ assmann-Wachmann 3 & 0.941 & 3.063 & 0.693 & 11.4 & 69.9 & 198.8 & 5.186 & 5.36 & $\tau$ Herculids \\
\hline $109 \mathrm{P} / \mathrm{Sw}$ ift-Tuttle & 0.958 & 26.317 & 0.963 & 113.4 & 139.4 & 153.0 & 51.675 & 135.00 & Perseids \\
\hline
\end{tabular}

Table 1. Orbital elements of 15 NEOs for which the responding, artificial streams have been generated. The orbits were gathered from Marsden and Williams (2003), NeoDys (2007).

element space, operating on a set of orbits $\mathbf{O}_{i}, i=1, \ldots, N$, was given by

$$
\rho\left(\mathbf{O}_{A}\right)=\sum_{i=1}^{N}\left(1-\frac{D^{2}\left(\mathbf{O}_{i}, \mathbf{O}_{A}\right)}{D_{c}^{2}}\right)
$$

- $W 2 D S H$ - a simplified Welch's method with $D_{S H}$ function, however the density at mean orbit of a stream was calculated from

$$
\rho\left(\mathbf{O}_{A}\right)=\sum_{i=1}^{N}\left(1-\frac{D\left(\mathbf{O}_{i}, \mathbf{O}_{A}\right)}{D_{c}}\right)^{2}
$$

- $W 1 D V$ - similarly to $W 1 D S H$, but with $D_{V}$ function as described in Jopek et al. (2008),

- $W 2 D V$ - similarly to $W 2 D S H$, but with $D_{V}$ function,

- $M I$ - method of indices (Svoreň et al. (2000)),

- $S L D S H$ - single neighbour linking technique with $D_{S H}$ function,

- $S L D V$ - as above, but with $D_{V}$ function.

All methods, except for the last three, were applied with the values of $D_{c}$ corresponding to their largest reliability. In case of $S L D S H$ and $S L D V$ methods, the constant threshold $D_{c}=0.02$ and $D_{c}=0.01 \cdot 10^{-2}$ were adopted. $M I$ method was used in the form described by Svoreň et al. (2000).

To evaluate the reliability level of the result obtained for a given stream with the applied method, we introduced two parameters, $S_{1}$ and $S_{2}$, defined as

$$
S_{1}=\frac{N_{p}}{N_{\max }} \cdot 100 \%, \quad S_{2}=\frac{N_{i}}{N_{p}+N_{i}} \cdot 100 \%
$$

where $N_{p}$ - a number of correctly identified members of a stream, $N_{\text {max }}$ - a total amount of particles in the stream, and $N_{i}$ - a number of interlopers i.e. sporadic meteoroids and meteoroids belonging to another streams.

In addition, for all streams identified at the same epoch, a general reliability parameter was evaluated with

$$
S S_{1}=\sum_{k=1}^{N} N_{k p} \cdot\left[\sum_{k=1}^{N} N_{k \max }\right]^{-1} \cdot 100 \%
$$

where $k=1, \ldots, N=15$, denotes all the identified streams. 


\section{Results}

At each epoch, for each stream, the values of the above parameters have been calculated. The results of the stream identification were accepted as reliable, only if $S_{2}<10 \%$. Next, a ranking of all reliable results was accomplished, and the results obtained are given in table 2 . We can see that the results with highest reliability were most often obtained using $D_{V}$ function and the Welch iterative or single linkage cluster analysis algorithm. When the same algorithms were used with $D_{S H}$ function, the results were considerably worse.

The ranking, in the way it was carried out, informs us only which of the methods was better. To illustrate the relative differences between the results obtained with various methods we need another measure. For this purpose we used a general reliability level $S S_{1}$. Close to the starting epoch $S S_{1}$ was above $90 \%$ for all the methods (Fig. 1), while as the stream dispersion proceeded in time the reliability of methods decreased. For $W 1 D V, W 2 D V$ and $S L D V$ methods decrease was approximately linear, while for $W 1 D S H, W 2 D S H, M I$ and $S L D S H$ methods the decrease of reliability was faster, with distinct fluctuation. In the first group the most reliable results were obtained more often with $W 1 D V$ method (from $100 \%$ to $60 \%$ ), and the reliability of $W 2 D V$ and $S L D V$ was equivalent. At the beginning, $S L D V$ was slightly more effective, while the $W 2 D V$ method gained the advantage in the later epochs. In the case of the second group of methods, their initial high reliability distinctly decreased with time, and finally reached values below $50-40 \%$. The lowest reliability, about $20 \%$, was obtained by $W 2 D S H$ method.

Table 2. Final score of the ranking of the meteoroid stream identification. In each column we see how many times a given method achieved the best result, e.g. at starting epoch, using $W 1 D V$ method three streams have been identified with the highest reliability level. Sometimes a few methods achieved exactly the same reliability for a given stream. In such cases each method scored one point.

$$
\text { Epoch } \backslash \text { Method W1DSH W2DSH W1DV W2DV MI SLDSH SLDV }
$$

\begin{tabular}{rrrrrrrr}
\hline 00000 & 2 & 2 & 3 & 3 & 10 & 8 & 4 \\
01200 & 2 & 1 & 1 & 2 & 3 & 1 & 8 \\
07200 & 0 & 0 & 7 & 3 & 2 & 0 & 5 \\
15200 & 0 & 0 & 10 & 3 & 0 & 0 & 3 \\
22000 & 0 & 0 & 2 & 5 & 2 & 0 & 4 \\
30000 & 0 & 0 & 10 & 6 & 0 & 0 & 0 \\
\hline Total & 4 & 3 & 33 & 22 & 17 & 9 & 24 \\
\hline
\end{tabular}

\section{Conclusion}

Our survey was the first step in the assessment of reliability of meteoroid stream identification methods. The obtained results let us state that identification methods based on $D_{V}$ function clearly distinguish themselves from others. Cluster analysis algorithms: simplified Welch's algorithm and single linking technique with $D_{V}$ function, most often appeared to be the most effective, whereas methods with $D_{S H}$ criterion, i.e $W 1 D S H$, $W 2 D S H$ and $S L D S H$ were less effective. 


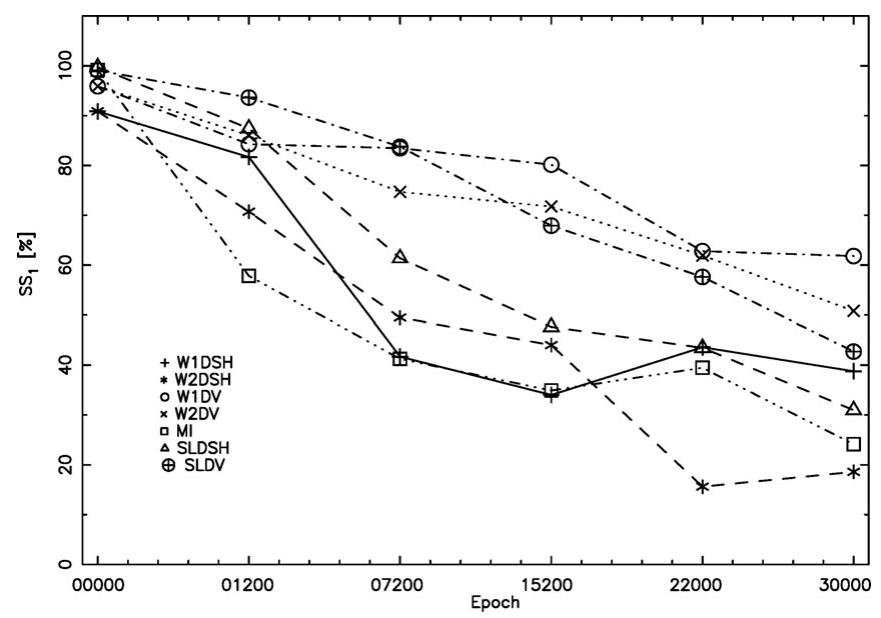

Figure 1. The general reliability parameter $S S_{1}$ obtained for all identified streams. The value of $S S_{1}$ was calculated by formulae 3 for each epoch.

\section{Acknowledgements}

The work was supported by the KBN Project N N203 2006 33. The majority of calculations were done at Poznań Supercomputing and Networking Centre. The authors wish to thank Dr. Anna Marciniak for improving English in the manuscript.

\section{References}

Brown, P., Weryk, R. J., Wong, D. K., \& Jones, J. 2008, Icarus, 195, 317

Drummond, J. D. 1981, Icarus, 45, 545

Galligan, D. P. \& Baggaley, W. J. 2002, IAU Colloq.181: Dust in the Solar System and Other Planetary Systems, 42

Jenniskens, P. 2008, Icarus, 194, 13

Jopek, T. J. 1993, Icarus, 106, 603

Jopek, T. J., Rudawska, R., \& Pretka-Ziomek, H. 2006, MNRAS, 371, 1367

Jopek, T. J., Rudawska, R., \& Bartczak, P. 2008, Earth, Moon and Planets, 102, 73

Lindblad, B. A., Neslušan, L., Porubčan, V., \& Svoreň, J. 2003, http://www.ta3.sk/ ne/IAUMDC/Ph2003/

Lindblad, B. A. 1971, Smithson. Contr. Astrophys, 12, 1

Marsden, B. G. \& Wiliams, G. V. (2003) Catalog of Cometary Orbits 2003, 15th edn, Smithsonian Astrophysical Observatory, Cambridge,MA

NEO Dynamic Site: 2007, http://newton.dm.unipi.it/neodys/

Southworth, R. B. \& Hawkins, G. S. 1963, Smithson. Contr. Astrophys, 7, 261

Sekanina, Z. 1976, Icarus, 27, 265

Svoreň, J., Neslušan, L., \& Porubčan,V. 2000, Planet. Space Sci., 48, 933

Valsecchi, G. B., Jopek, T. J., \& Froeschle, C 1. 1999, MNRAS, 304, 743

Welch, P. G. 2001, MNRAS, 328, 101 